\title{
Prostate Cancer: Importance of Screening and Early Diagnosis
}

\author{
*MT Rahman
}

\section{Introduction}

Adenocarcinoma of prostate is the most common form of cancer in men $(25 \%)$ by incidence and second $(10 \%)$ by mortality throughout the world. There is one in six life time possibility of being diagnosed with prostate cancer in men. Prostate cancer is a disease of men over 50 years of age. In autopsy the incidence of prostate cancer is very high $20 \%$ in 50 years age and $70 \%$ between $70-80$ years. According to a survey in 2014 about 3,00,000 prostate cancer patients die every year, 1.1 million new cases are diagnosed.

An early diagnosis and preventive measures could lower this mortality. What preventive measures and diagnostic tests could be advised for prostate cancer? We know risk factors for prostate cancer includes obesity, increased testosterone level, increasing age, family history, genetic predisposition, lack of exercise, lower level of vitamin D and STD like Chlamydia, gonorrhea and syphilis, prostatitis increases the risk of prostate cancer while lipid lowering drugs like statins, exercise, low testosterone level, antioxidant, safe sex, Normal BMI screening of family members of affected cancer patients, maintenance of normal blood pressure lowers the risk of prostate cancer. So if we could follow this simple preventive measures we could reduce the mortality from prostate cancer.

Early screening and diagnosis is very important for reducing morbidity and mortality of prostate cancer. It is already known and mentioned in the literature that the diagnostic triad for prostate carcinoma include USG of prostate, PSA level estimation and biopsy of prostate is sufficient for diagnosis of majority of prostate cancer cases.

PSA estimation should be done every three years for male over 50 years of age. Although normal value upto $4 \mathrm{ngm} / \mathrm{dl}$ if PSA level is $2 \mathrm{ngm} / \mathrm{dl}$ in three consequitive sample within three months it is indicative of negative for malignancy. Gray Zone 4$20 \mathrm{ngm} / \mathrm{dl}$ and over values can be seen in other conditions apart from prostate cancer. These include Benign Hyperplasia Prostate, Prostatitis, Instrumentation, ejaculation, riding bicycle, drugs like testosterone, procedures like catheter, biopsy, cystoscopy.

Other types of newer PSA tests like free and total PSA ratio is very sensitive and diagnostic. If the free and total PSA ratio is above 20 it is indicative of benign condition and if the ratio is less than 10 it is indicative of malignant lesion. PSA velocity level goes faster in man having prostate cancer and normal PSA velocity goes slower with age. PSA density is also an important tool in prostate cancer diagnosis. If prostate density is higher it indicates a likelihood of prostate cancer. USG is an important tool in the early diagnosis of prostate cancer. because it can detect both velocity and density.

Biopsy either TURP, Core or Gun, needle biopsy is very important in diagnosis and staging of prostate cancer histopathology using combined Gleason score and TNM classification is essential for management of prostate cancer. PSMA is a tumour marker for early detection of metastasis and ER/PR is also important tumour marker for prognosis of prostatic cancer. Genetic studies like BRCA1,2, HPC1, SNP specially with TT allele pair at SNPrs10993994 is indicative of important increased risk of prostate cancer development.

There is no specific sign and symptoms of prostate cancer. However studies have shown that about one third of patients diagnosed with prostate cancer had one or more of the following sign and symptoms. These include frequency, nocturia, urgency, hesitancy, hematuria, dysuria, UTI, difficulty and

Prof. Dr. Md. Tahminur Rahman, Visiting Professor of Pathology, Anwer Khan Modern Medical College

e-mail: mtahminur@yahoo.com

*Corresponding Author

AKMMC J 2019; 10(1) : 03-04 
pain full ejaculation, erection although these symptoms are also found in BHP. Metastatic prostate cancers have additional symptoms. The most common symptom is bone pain often in spine, pelvis, ribs. Prostate cancer can also compress the spinal cord causing tingling, leg weakness urinary and fecal incontenance. So if these symptoms are manifested the patient should consult urologist immediately and do the investigations mentioned above.

In conclusion it can be said that simple preventive measures mentioned before, expression of two or more sign and symptoms, Bone pain, Weakness, tingling of legs should be immediately brought notice of your doctor and the patient should undergo for USG, PSA, PRDE, Biopsy, tumour markers for exclusion of diagnosis of prostate cancer. These will definitely define better strategies and management of prostate cancer and reduce morbidity and mortality in prostate cancer patients. The early we awake the better the outcome.

\section{References}

1. Robbins and Cotran Pathologic Basic of Disease, Editor Kumar, Abbas, Fausto, Aster $8^{\text {th }}$ edition, 2010, ELSEVIER Saunders India, Ltd.

2. Rahman MT, Mumu, MA, Kabir, Chowdhury MM, et al. Serum Zinc and Different Prosatatic Lesions. Mymensingh Med J 2012 OCT, 21(4): 679-6873.

3. Rahman MT at al. Serum Zinc, Total \& Free PSA, Free/Total PSA ratio and prostate cancer Associaiton: A case control study. AKMMCJ 2013; 4(1): 15-19.

4. Rahman MT, Chowdhury ATMM. Prostate cancer. Review article. AKMMCJ 2016; 7(2): 36-44. 